\title{
$\mathrm{X}$.
}

Aus der Marburger chirurgischen Klinik.

Direktor: Geb. Medizinalrat Prof. Dr. Kü ster.

\section{Ein Beitrag zur Kenntnis der traumatischen Luxationen des Fufses im Talocruralgelenke. \\ von}

Dr. med. Johannes Richter,

ehem. Volontairassistenten der Klinik.

(Mit 2 Abbildungen).

Die Anregung, mich mit dem beutigen Stande der Wissenschaft auf dem Gebiete der traumatischen Fußluxationen im Talocruralgelenke etwas eingehender zu befassen, verdanke ich einem Falle, den ich unlängst in der Marburger chirurgischen Klinik zu beobachten Gelegenheit batte.

Für gütige Überlassung desselben zur Veröffentlichung und liebenswürdige Durchsicht meiner Arbeit spreche ich Herrn Geheimrat $\mathrm{K}$ ü ster hiermit meinen verbindlichsten Dank aus; ebenso fühle ich mich Herrn Prof. Enderlen für das mir hierbei bewiesene liebenswürdige Entgegenkommen sehr zu Danke verpflichtet.

Die Krankengeschichte des Falles ist folgende:

Unvollkommene Verrenkung des Fußes nach hinten mit Bruch der Fibula. Leichte Reposition. Vollständige Heilung.

Am 2. Juli d. J. gelangte die 18 jährige, bisher stets gesunde Landarbeiterin E. M. zur Aufnahme in die Klinik mit der Angabe, sich am genannten Tage bei der Feldarbeit vor etwa 2 Stunden eine Verletzung des linken Fußes folgendermaßen zugezogen zu haben:

Bei dem Versuche, von einem mit einem Fuder Klee beladenen Wagen herabzusteigen, sei sie aus etwa $1 \frac{1}{2} \mathrm{~m}$ Höhe derart abgestürzt, daß sie mit der Vorderseite des Körpers langgestreckt auf den Boden zu liegen kam. Der linke Fuß sei dabei nach außen gedreht gewesen. Sie habe sofort die Empfindung gehabt, als sei das linke Bein in der Fußgelenkgegend ge brochen, habe sich nicht mehr aufrichten können und sei alsbald ohne vorherige Hinzuziehung eines Arztes zur Klinik geschafft worden.

Status: Kräftig gebautes, gesund aussehendes Mädchen mit mäßiger 
Kropfanlage, innere Organe gesund. Der linke Unterschenkel im unteren Drittel nebst Fußgelenkgegend erheblich geschwollen. Am Fußrücken in der Gegend des Talocruralgelenkes ein bogenförmiger Vorsprung von knöcherner Resistenz dicht unter der Haut wahrnehmbar. Der Fuß steht in leichter Plantarflexion und mäßiger Valgusstellung. Er ist nicht ganz unbeweglich in dieser Stellung fixiert, läßt jedoch nur ganz leichte Beugung und Streckung im Talocruralgelenke zu. Eine genauere Orientierung über den Knöchelstand ist wegen der bestehenden Anschwellung nicht möglich. Es besteht am medialen Knnöchel keine auffallende Druckempfindlichkeit, wohl aber ist ausgesprochener Druckschmerz etwa $8 \mathrm{~cm}$ oberhalb der Knöchelspitze an der Fibula vorhanden, woselbst auch Crepitation nachweisbar ist. Die Durchleuchtung mit Röntgenstrahlen zeigt, daß die Tibia sich mit ihrem unteren Ende nach vorn verschoben hat und am Fußrüeken einen deutlichen Vorsprung bildet. Sie ist jedoch nicht gänzlich außer Contact mit der Talusrolle geraten, sondern berïhrt mit ihrer Gelenkfläche noch ein wenig den vorderen Teil der correspondierenden Talusgelenkfläche. Die Fibula ist ca. $8 \mathrm{~cm}$ oberhalb der Knöchelspitze gebrochen; das untere Fragment, welches mit dem oberen einen nach hinten offenen Winkel bildet, befindet sich im Zusammenhange mit dem Talus und ist an letzterem mitsamt dem ganzen Fuße nach hinten verschoben. Dementsprechend ist der Vorderteil des Fußes verkürzt, der Fersenteil verlängert. Die Diagnose lautet dementsprechend: unvolllkommene Luxation des Fußes im Talocruralgelenke nach hinten mit Fractur der Fibula. Am 4. Juli 1904 wird in Narkose durch Zug am Fuße in der Längsachse des Beines und gleichzeitigen Druck in der Richtung von vorn nach hinten auf das untere Tibiaende die Reposition mit Leichtigkeit vollführt, das Bein zunächst auf Volk$m$ ann scher Schiene fixiert.

11. Juli. Fuß in guter Stellung. Gypsverband.

20. Juli. Neuer Gypsverband.

21. Juli. Patientin steht auf.

4. August. Gypsverband entfernt, Stellung gut, Massage.

7. August. Leichte medicomechanische Übungen. Das Gehvermögen mit jedem Tage besser.

13. August. B ef und: Linker Untersehenkel im unteren Drittel etwas verdickt, nach längerem Geben kommt es noch zur Anschwellung. Die Beweglichkeit des Fubgelenkes noch etwas eingeschränkt, die Fibulafractur ist fest verbeilt. Ohne Röntgendurchleuchtung ist keine Dislucation sichtbar; mit dieser läßt sich eine kleine Abweichung des unteren Fragmentes nach hinten erkennen, die jedoch der Patientin nicht nachteilig ist. Der Talus befindet sich an richtiger Stelle. Die Muskulatur beider Unterschenkel annähernd von gleichem Unfange. Der Gang ohne Stock hinreichend sicher und flott. Patientin wird auf ihren Wunsch entlassen.

Fassen wir die Krankengeschichte kurz zusammen, so handelt es sicb bei einem gesunden, kräftig gebauten Mädehen um Entstehung einer Fußbezw. Unterschenkelverletzung durch Fall vornüber aus nicht erheblicher IIohe mit Aufschlagen auf den Boden in gestreckter Körperhaltung. Die an demselben Tage in der Klinik vorgenommene Untersuchung ergibt vor der Röntgendurchleuchtung wegen erheblicher Anschwellung der Gelenkgegend kein ganz sicheres Resultat, indem sich mit Bestimmtheit nur die 
Fibulafractur erkennen läßt. Erst durch das Röntgenbild wird die Dia-gnose der Fußgelenkluxation sichergestellt, und es werden dadurch gleichzeitig die Einzelheiten der complicierenden Fibulafractur, die schon vor der Röntgendurchleuchtung mit Bestimmtheit diagnosticiert war, sichtbar gemacht; auch zeigt es sich, daß die Luxation keine vollkommene (complete) ist. Die Reposition gelingt in Narkose mit Leichtigkeit; der weitere Heilungsverlauf war ein glatter. Nach gut 5 Wochen kann Patientin bereits als wesentlich gebessert mit guter Function entlassen werden.

Soweit unser Fall.

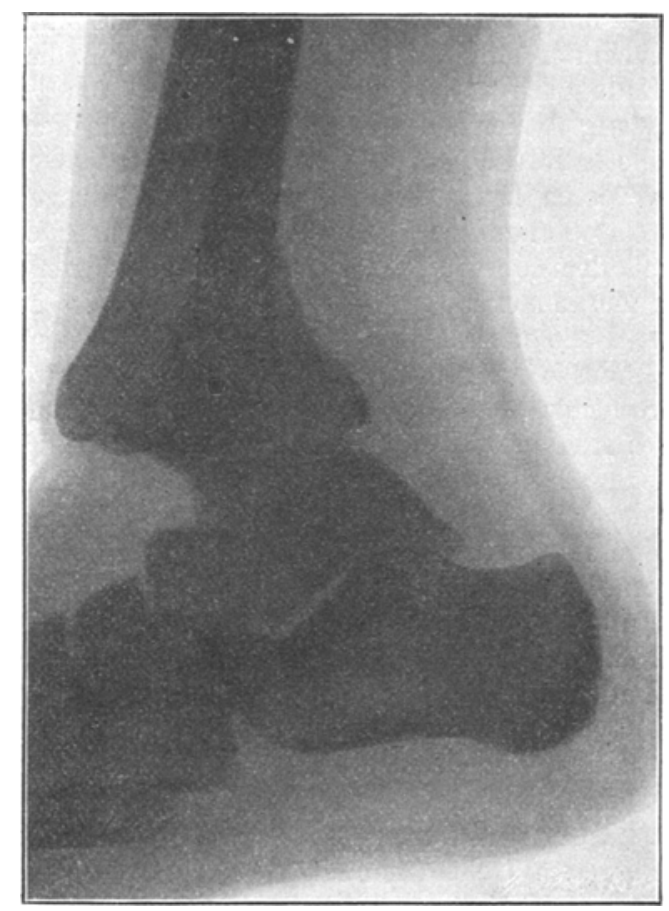

Fig. 1.

Bei Durchsicht der ziemlich spärlichen Literatur nach den bisherigen Veröffentlichungen von Luxationen des Fußes (in Talocruralgelenke) nach hinten bin ich auf eine einzige umfassende Zusammenstellung der bis jetzt bekannten Fälle gestoßen. Dieselbe ist in der Arbeit von Wendel (1) 1) (aus der Tübinger chirurgischen Klinik) über die traumatischen Luxationen des Fußes im Talocruralgelenke ent-

1) Die Zahlen beziehen sich auf das am Schlusse dieser Arbeit befindliche Literaturverzeichnis. 
Ein Beitr. zur Kenntnis d. traumat. Luxationen des Fußes im Talocruralgel. 249

halten. In dieser Abhandlung sind 25 Fälle aus der Literatur angeführt, und diesen ist ein von Wendel selber beschriebener neuer Fall hinzugefügt mit dem Bemerken, daß diese 26 Fälle sämtlich reine, d. h. von keiner Fractur begleitete Luxationen des Fußes nach hinten seien. Der neuste der Wendelschen Fälle datiert vom Ende des Jahres 1896 und ist ebenso wie die übrigen genannten Fälle nicht mit Röntgenbild versehen.

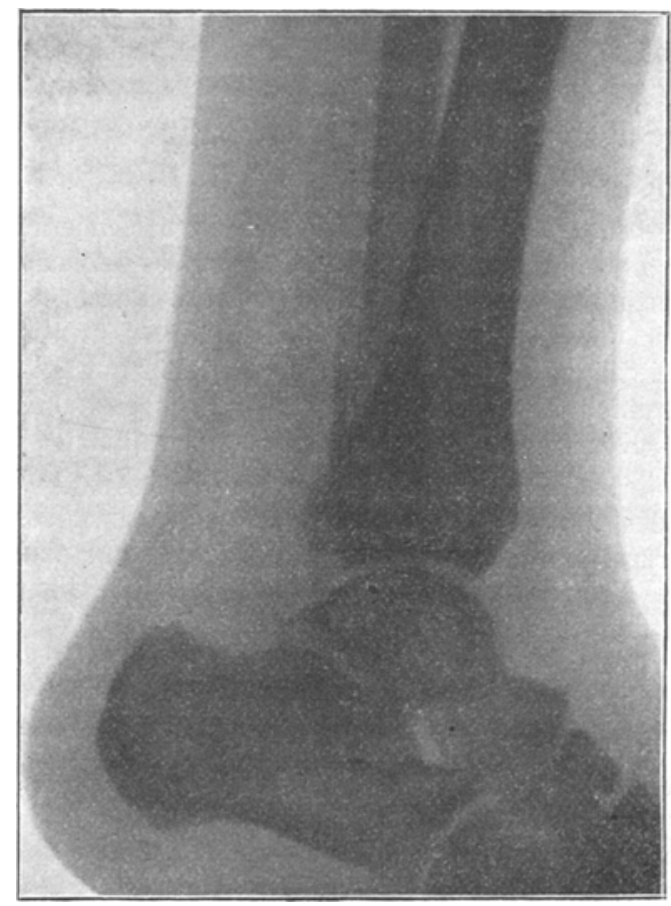

Fig. 2.

Angeregt durch die Wendelsche Arbeit habe ich versucht, in der Literatur hierher gehörende sonstige Fälle neuester Beobachtung aufzufinden; es ist mir gelunigen vom Jahre 1896 ab bis jetzt (December 1904) 7 Fälle der Art zusammenzustellen, von denen die 5 jüngsten mit Röntgenaufnahmen versehen sind.

Ich lasse die Krankengeschichten der ehronologisch geordneten Fälle jetzt folgen.

1. Göschel (2).

59 jähriger Herr ging im Dunklen eine Wendeltreppe herunter, er glitt aus, fiel nach rückwärts und rutschte dann mit den Füßen voraus etwa 
6 Stufen herab, bis er mit den Füßen gegen einen Lattenverschlag anstieß. Er konnte nicht mehr aufstehen. Ein anderer hatte ihn aufgehoben, seinen nach außen stehenden $F u ß$ hereingedreht und ihn dann nach Hause geschafft. Dort bot sich folgendes Bild: linker FuB gering plantarflectiert, in Mittelstellung, die Ferse weit nach hinten gedrängt. Vorn am Sprunggelenk springt die Tibia weit vor, so daß ihre untere Kante und ein Teil der Gelenkfläche abzutasten ist. Eine Knöchelfractur kann nicht nachgewiesen werden. Reposition in Narkose durch kräftige Plantarflection, Druck auf das untere Tibiaende nach hinten, Druck auf die Ferse in der Richtung nach vorn. Unter lautem Geräusch verschwand die Difformität. 8 Tage Gypsverband, dann Massage.

2. G ömöry (3). Ein Einjährig-Freiwilliger stürzte am 4. Januar 1902 in einem Mannschaftszimmer der Brïner Landwehrkaserne zu Boden. Der Sturz kam auf die Weise zustande, daß der Freiwillige vom Gange her in das Zimmer lief, dessen Tür offen stand, wobei er ungefähr drei Schritte von der Tür entfernt gegen einen dort stehenden Kameraden, den er zu spät bemerkte, anrannte und mit |seinem rechten Fuße von rückwärts zwischen die beiden Füße desselben gelangte. Teils durch den heftigen Anprall, teils durch das Fixieren seines rechten Fußes zwischen den Füßen des anderen Freiwilligen verlor er das Gleichgewicht und stürzte mit großer Gewalt rechts seitlich zu Boden, worauf sein Kamerad ebenfalls hinstürzte und mit seinem Körpergewichte auf den Unterschenkel des am Boden Liegenden auffiel.

Während des Sturzes und unmittelbar nach demselben hatte Patient keinerlei Schmerzempfindungen, sondern nur das Gefühl eines in den Schuh hineingeglittenen Fremdkörpers, „als ob ihm das Taschenmesser aus der Hosentasche in den Schuh hineingerutscht wäre". Dabei griff er tatsäehlich nach dem Fuße, um sich zu überzeugen, ob das Taschenmesser sich wirklich im Schuh befinde.

Der 2 Stunden danach im Spital erhobene Befund war folgender:

Der Rücken des rechten Fußes ist verkürzt, die Ferse verlängert. Nach vorn und etwas nach innen springt die Gelenkfläche der Tibia am Fußrüeken weit vor und kann durch die Haut sehr deutlich abgetastet werden. Die scharf markierten Conturen des vorderen Randes der leeren Tibiapfanne gehen nach innen in den ebenfalls sehr deutlich vortretenden Malleolus internus über. Gegen den inneren Knöchel nnd die mediale Hälfte der Tibiapfanne zieht die gespannte Haut von der Innenseite der Ferse und dem Innenrande des Fußes teils in gröberen Erhebungen, teils in kleineren Furchen und Falten hin. Die Strecksehnen des Fußes, namentlich die zur großen Zehe ziehenden, sind in ihrem Verlaufe über den Fußrücken als strangartige, die Haut von der Unterlage etwas abhebende Gebilde zu sehen. Der äußere Knöchel ist mit der Ferse nach hinten und etwas nach außen abgewichen und erscheint die Fibula $6 \mathrm{~cm}$ oberhalb der Malleolenspitze stumpfwinklig geknickt. An der Spitze dieses Winkels ist Crepitation nachweisbar und ein spitzes Knochenfragment zu fühlen. Das Gelenk erscheint im Querdurchmesser stark verbreitert. Die Achillessehne zieht gespannt in einem nach hinten concaven Bogen zur Ferse. Zwischen ihr und der Tïbia kann man in eine flache Hohllrinne greifen, fibularwärts nicht. Der Fuß 
Ein Beitr. zur Kenntnis d. traumat. Luxationen des Fußes im 'Talocruralgel. $2 \vdots 1$

im ganzen ist plantarflectiert und um seine verticale Achse (um ca. 700) nach außen rotiert.

Weichteilschwellungen und Blutergüsse fehlten gänzlich, so daß die Diagnose nicht schwierig war. Vor der Reposition wurde ein Gypsabguß des Fußes hergestellt.

Die Reposition gelang durch leichten $\mathrm{Zug}$ am Fuße ohne alle Schwierigkeit, alsdann Fixation der Extremität 6 Wochen im Contentiv-Verbande (zuerst Gyps-, während der letzten 3 Wochen Organtin-Verband). Schon in der 2. Woche Gehversuche, nach den ersten 3 Wochen bereits Consolidation. Nach Ablanf von 6 Wochen bestand eine nennenswerte Verdikkung des Gelenkes nicht, das letztere zeigte normale Umrisse. Die passive Beweglichkeit des Gelenkes ist eine gute. Die Nachbehandlung bestand in täglicher Massage, passiven Bewegungen, zeitweiser Anwendung warmer Bäder, vorsichtiger Aufnahme der Gehversuche mit allmählicher Steigerung der Gelenkfunctionen. Hiernach ist eine vollkommene Restitutio ad integrum vorauszusehen.

3. Schanz (4).

45jährige Frau trat am 4. Mai 1902 in S.'s Behandlung (orthopädische Heilanstalt Dresden); sie gab an, im November 1901 verletzt zu sein. Sie sei infolge Strauchelns eine kleine Treppe herabgerutscht und am Fuße derselben so zu Falle gekommen, daß sie sich auf den untergeschlagenen linken Fuß setzte. Der Fuß sei mit der Spitze hängen geblieben und dadurch in Plantarflexion geraten. Sie wurde vom Platze getragen. Der Arzt constatierte eine Verstauchung des Fußgelenks und legte eine Volkmannsche Schiene an. Nach einigen Wochen begann die Patientin wieder zu gehen, der Fuß blieb aber deform, seine Function blieb eine recht mangelhafte.

Der durch Röntgenphotographie illustrierte Befund S.'s ist folgender: Besonders fällt die Vergrößerung des anterio-posterioren Durchmessers des Fußgelenkes auf. Die Fußspitze zeigt nach auswärts. Vom Fußrücken her kann man mit der Fingerspitze unter die Gelenkfläche der Tibia hereindrücken. "Der äuBere Knöchel ist nicht luxiert." Oberhalb desselben finden sich die Spuren einer Fractur der Fibula. Diese Fractur ist auf der Röntgenphotographie deutlich zu erkennen. Das Aktinogramm zeigt, daß die Gelenkfläche der Tibia an der des Talus nach vorn abgeglitten ist. Es scheint vom innern Knöchel ein Stück abgebrochen und im ursprünglichen Zusammenhange geblieben zu sein. Die Beweglichkeit des Fußgelenkes ist etwa um die Hälfte eingeschränkt. Der Gang der Patientin läßt viel zu wünschen übrig. Sie benutzt einen Stock und hinkt erheblich. Sie klagt über Schmerzen beim Gehen und beim Stehen. Der Versuch, in Narkose die Luxation zu reponieren, gelang nicht. Es wurde dann der Patientin ein Schienenhülsenapparat gegeben. Die damit erzielte Functionsbesserung war so erheblich, daß auf eine blutige Reposition verzichtet werden konnte.

4. Engelhardt (5).

Am 6. Juli 1902 wurde in die Gießener chirurgische Klinik ein 18. jähriger Patient eingeliefert, der im maschinellen Betriebe bei dem Versuche, von einer in $3 / 4 \mathrm{~m}$ über dem Boden befindlichen, mit großer Geschwindigkeit rotierenden Drehscheibe den Treibriemen abzunehmen, wahrscheinlich 
mit der rechten Hand zwischen Riemen und Scheibe geriet und mit großer Gewalt gegen die etwa $3 / 4 \mathrm{~m}$ entfernte Wand geschleudert wurde, wo er eine halbe Stunde bewuftlos liegen blieb. Ob sein rechter $F u ß$ dabei in irgend einer Stellung fixiert, der Körper nach einer anderen Richtung herumgerissen wurde, weiß er nicht anzugeben. Der Befund am Tage nach der Verletzung war folgender:

Der rechte Fuß ist im Bereiche des Talotibialgelenkes stark geschwollen, ebenso der rechte Unterschenkel. Aktive Bewegungèn sind im Gelenke nicht möglich, passive nur in ganz geringem Grade und mit großen Schmerzen verbunden. Bei näherer Untersuchung findet sich vor dem Gelenke quer verlaufend eine tiefe Furche, die durch eine Vorwölbung der Haut durch einen deutlich palpierbaren stumpfen Knochenrand, der dem untern Gelenkende der Tibia entspricht, bedingt ist. Bei rechtwinkliger Stellung des Fules überragt das untere Tibiaende den vordern Talusrand um etwa $21 / 2 \mathrm{~cm}$, während der Abstand des hintern Tibiarandes vom Ansatz der Achillessehne an den Calcaneus verlängert ist. Die Achillessehne selbst verläuft in leicht convexem Bogen zu ihrer Ansatzstelle. Eine Verschiebung der Tibia nach innen ist nicht $\mathrm{zu}$ constatieren. Die Fibula findet sich an normaler Stelle in fester Bandverbindung mit dem Fuß. Druck auf den äußeren Knöchel ist nicht schmerzhaft

Eine sogleich von vorne und von beiden Seiten aufgenommene Röntgen photographie bestätigte die Annahme einer Luxation des Fußes mit der Fibula nach hinten. Am Malleỏlus internus findet sich eine kleine Abrißfractur, die Fibula ist laut Röntgenbild in ihrer ganzen Länge intact, das obere Ende der Fibula steht an normaler Stelle. Die in Chloroformnarkose vorgenommene, durch Plantarflexion, Extension und spätere Dorsalflexion bewirkte Einriehtung gelang nur schwer und nieht vollkommen. Wegen der hochgradigen Schwellung wurde zunächst kein Gypsverband angelegt, sondern das Bein nur auf Volkmannscher Schiene gelagert. Der weitere Verlauf war durch Vereiteruug eines wahrscheinlich bei der Reposition entstandenen Blutergusses mit hohem Fieber compliciert, wodurch auch in der nächsten Zeit die Anlegung eines circulären Gypsverbandes unmöglich wurde. Patientin kam nach 8 Wochen mit fester Ankylose in guter Stellung des Fußes zur Entlassung. Das Heilungsresultat wurde nachträglich etwas verschlechtert durch das Hinzutreten einer ganz geringen Spitzfußund Supinationsstellung. Eine am 10. Oktober vorgenommene Durchleuchtung zeigt an der Vorder-und besonders an der Rückseite des Gelenkes diffuse Schatten von neugebildetem Knochen herrührend. Es besteht noch eine hochgradige Atrophie des Beines. Doch kann Patient das Bein zum Auftreten und Gehen gut benutzen.

5. Nasse und Burchardt: Fall aus der v. Bergmannsehen Klinik (17).

Junge Frau hat sich, in typischer Weise dureh einen Fall bei extremer Plantarflexion des Fulses eine Luxatio pedis nach hinten zugezogen. Der Fuß war in der pathologischen Stellung absolut fixiert; eine Fractur der Fibula ließ sich palpatorisch nicht nachweisen, trotzdem war sie wie das Röntgogramm zeigt, vorhanden."

6. Füster (6). Fall 1.

Am 23. Mai 1904 wurde eine 59jährige, sonst stets gesunde Frau in die Grazer chirurgische Klinik aufgenommen. Dieselbe war 9 Wochen vor 
ihrer Aufnahme auf glattem Erdboden ausgerutscht und mit voller Wucht nach rückwärts gefallen, wobei gleichzeitig ihr rechter $\mathrm{Fu} B$ in einen glattwandigen, aus festem Material bestehenden Kanal so zu liegen gekommen war, daß die Planta pedis bei sehräger Haltung des Fußes, das heißt beim Anstemmen desselben gegen die starre Wand des Kanals, an diese letztere mit starker Wucht anprallte. Die Frau konnte sich nach dem Falle nicht mehr erheben und hatte heftige Schmerzen im rechten Fuße; ein herbeigerufener Arzt applicierte wegen der rasch aufgetretenen Schwellung in der Gegend des rechten FuBgelenkes ihr zuerst feuchte Umschläge, dann soll sich angeblich zu einer dortselbst befindlichen, infolge des Sturzes entstandenen Hautwunde ein Erysipel hinzugesellt haben, nach dessen Abheilung einer bestehenden Unterschenkelfractur halber der Frau ein Gypsverband bis unter das Knie reichend angelegt wurde und 7 Wochen lang liegen blieb. Nach Abnehmen desselben resultierte einerseits eine fortbestehende Deformität, andererseits ist die Beweglichkeit des rechten Fusses eine dauernd hochgradig eingeschränkte. Da auberdem die in Massage und warmen Bädern bestehende Nachbehandlung keinen Erfolg herbeiführte, suchte die Frau die Klinik auf.

A ufnahmebefund: Bei der sonst gesunden Frau, die sich nur mühsam mit Hilfe zweier Stöcke kleine Strecken fortbewegen kann, ist der rechte $\mathrm{Fu}$ in in seinem vorderen Abschnitt scheinbar verkürzt und zeigt in der Gegend des Talocruralgelenkes, dessen aktive Beweglichkeit vollständig aufgehoben ist, folgende Veränderungen: Zunächst sieht man dort eine markante Vorwölbung, gebildet durch den deutlich abtastbaren glatten Rand der Gelenkfläche der Tibia. Oberhalb dieser Stelle befindet sich ein circa 1 cm langer, $1 / 2$ cm breiter, die Haut durchgreifender Substanzverlust. Dort, wo durch obige Vorwölbung eine Stufe gebildet wird, ist die Haut nicht vollständig straff gespannt, sondern etwas faltig.

Die Achillessehne verläuft in einem nach hinten konkaven Bogen zum Fersenhöcker herab und läBt zwischen sich und der Tibia eine tiefe Rinne frei. Der Tuber calcanei springt stark vor. Die Bewegungen im unteren Sprunggelenk sind in geringem Grade eingeschränkt. Der FuB steht in Plantarflexion, die Zehen werden etwas gebeugt gehalten. Ein deutliches Vorspringen der Strecksehnen am Fußrücken ist nicht konstatierbar. Die Palpation sowohl der Gegend des Malleolus externus als internus ist schmerzhaft, stärker ausgesprochen am äußeren Knöchel, Crepitation und abnorme Beweglichkeit von seiten der Fibula sind nicht nachweisbar. Die Diagnose der Luxatio pedis posterior wurde durch die Röntgenuntersuchung bestätigt und dahin ergänzt, daß eine Abrißfraktur am Malleolus internus mit Dislokation des Bruchstückes nach hinten vorhanden ist. Eine Fibulafractur läßt sich auch aus dem Röntgenbilde nicht konstatieren, aber auch nicht ausschließen.

Therapie: in Chloroformnarkose wird dureh eine Kombination von manuellem und instrumentellen Redressement unter Benutzung des Lorenzschen Osteoklastredresseurs die Reposition mit verhältnismäßig recht gutem Erfolge ausgeführt. - Eine Deformität ist danach äuBertich nicht mehr wahrnehmbar. Im Röntgenbilde läßt sich allerdings erkennen, daß die Stellung der Gelenkenden noch keine vollständig normale ist. Anlegung eines bis zum Tibiaknorren reichenden Gypsverbandes bei Dorsalhyperflexion des 
Fußgelenkes. Nach 12 Tagen Verbandwechsel. Anlegung eines leichteren Gypsverbandes (Wasserglas), mit welchem am Tage darauf bereits Gehversuche gemacht werden. Einige Tage darauf auf ejgenen $W$ unsch entlassen, gibt Patientin nach ca. 6 Monaten der Klinik die Nachricht, da 3 sie mit dem verletzten Fuße ebensogut wie mit dem gesunden gehe (an dem nur hier und da nach stärkeren Strapazen eine unbedeutende Schwellung in der Gelenkgegend auftrete) und daß sonst die Funktion desselben ebenso vollkommen normal sei wie vor der Verletzung.

7. Füster (6) Fall II. 59 jährige Frau stiirzte am Morgen ihrer Aufnahme in die Klinik eine Treppe herab, wobei ihr rechter Fuß unter ihren Körper zu liegen kam. Dabei soll der Fuß eine vorübergehende Hyperflexions- und Supinationsstellung eingenommen haben. Die Deutlichkeit des am Nachmittag desselben Tages sich darbietenden Bildes war durch die starke Schwellung der unteren Hälfte des rechten Unterschenkels sowie des Fußes beträehtlich verwischt; es ließ sich neben der typischen Bewegungseinsehränkung und sehr starken Schmerzhaftigkeit hauptsächlich ein auffallend starkes Vorspringen des Fersenhöekers nach hinten konstatieren und konnte bei Pround Supinationsbewegungen des Fußes manifeste Crepitation festgestellt werden, ohne daß man dieselbe aber einwandfrei und genau hätte lokalisieren können. Im Röntgenbilde sieht man, daß die Luxation nach hinten diesmal mit Abrißfracturen im Bereiche beider Malleolen kompliziert ist. Die beabsichtigte Reposition wurde wegen Widerspruches der Patientin nicht unternommen und letztere ungeheilt mit einem einfachen Schienenverbande entlassen.

Allgemeine Bemerkungen, Entstehungsinechanismus.

Gehen wir nunmehr zu einer allgemeinen Besprechung der Luxatio pedis posterior über, so zeigt eine Durchsicht der einschlägigen Literatur (und der modernen Lehrbücher), daß sie zunächst insofern eine bevorzugte Stellung im System der Fußgelenkverrenkungen einnimmt, als sie außer der Luxatio pedis anterior (mit der sie die Gruppe der sagittalen Fußgelenkluxationen bildet) die einzige ist, welche allgemein als echte - ohne vorausgegangene Malleolenfraktur mögliche Luxation des Talocruralgelenkes anerkannt ist.

Für die Mehrzahl der Autoren, so K oenig (7), Leser (8), A lbert (9), Helferich (10), Lossen (11), Gurlt (12) ist mit ihrer Besprechung: und derjenigen ihres Gegenstïcks (der Luxation nach vorn) das Kapitel der Talocruralgelenkluxationen erschöpft. Was ihr Vorkommen betrifft, so gilt sie auch heute noch als selten. Unter 400 frischen Luxationen der $\mathrm{Kr}$ önleinschen (13) Zusammenstellung aus der Berliner Klinik und Poliklinik finden sich nur 2 Luxationen des Fußes nach hinten (d. i. nur 0,5 Proz. aller Luxationen) bei einem Beobachtungszeitraum von 6 Jahren.

Wie das Verbältnis ihrer Häufigkeit zu derjenigen der übrigen 
Fußgelenkluxationen ist, läßt sich am besten aus Wend els (1) Arbeit ersehen, in der sich unter 108 Fufgelenkluxationen nur 26 solcher nach hinten beschrieben finden. Allerdings ist sie danach etwa doppelt so häufig als die vordere Luxation (nach Wendel 11 Fälle). Nicht ohne Interesse dürfte die Bemerkung sein, daß sie von $\mathrm{Du}$ puytren in 15 Jahren nur 2 mal beobachtet worden ist (citiert bei Gömöry (3)). Nach Abschluß der Wendelschen Arbeit sind nur 7 neue Fälle in der Literatur - soweit mir dieselbe zugänglich war - beschrieben worden, deren Krankengeschichten vorstehend angeführt sind.

Ebensowenig wie über ihren Charakter als echte primäre Fufgelenkluxation und über ihre Seltenheit bestehen erhebliche Meinungsverschiedenheiten der Autoren hinsichtlich ihrer Ätiologie.

Eine übermäßige Plantarflexion ist für das Zustandekommen derselben erforderlich und wird nach Lossen (11) in der Regel durch die Körperschwere erzeugt, welche bei fixiertem Fuß oder während derselbe auf eine nach vorn abschüssige Ebene auffällt, „den Unterschenkel im Rückwärtsfallen des Stammes nach hinten reißt." Diese Entstehungsart durch Rückwärtsfallen wird fast allgemein von den Autoren als typisch angegeben; sie ist auch nach den vorliegenden Beobachtungen die häufigere.

Ein besonders charakteristisches Beispiel hierfür ist $\mathbf{u}$. a. der Fall Noquet(Stoß mit dem Fuß gegen den Rand einesTrottoirs und Sturz nach hinten (cit. bei Wendel (1)), ferner der Fall Socins (Turner, der beim Sprunge über das Pferd beim Absetzen des rechten Fußes nach hinten fiel) (cit. bei Wendel) (1)), alsdann ein Fall Dupuytrens (Patientin blieb, als sie vom Schwindel befallen rückwärts stürzte, mit dem Fuße zwischen Tisch und Kamin hängen), cit. bei Lossen (14); ganz ähnlich der Fall Polaillons (Patientin blieb beim Fall von einer Leiter mit dem Fuße zwischen 2 Sprossen hängen), cit. bei $W$ endel (1)). Als Curiosum sei an dieser Stelle auch der Fall Wendels (1) erwähnt, wo einem Bauernburschen in einer Treibjagd ein Hirsch gegen den Unterschenkel sprang und so ein Rückwärtsfallen bewirkte.

Complieierter als in den eben genannten Fällen war der Vorgang bei Göschels (2) Patienten, der eine Wendeltreppe 6 Stufen mit den Füßen voraus herabrutschte, bis er mit den Füßen gegen einen Lattenverschlag anstieß. Hier bestanden zwei Componenten der Gewaltrichtung: einmal die von oben und hinten nach vorne und unten in der Richtung der Unterschenkelachse als Stoß wirkende Körperschwere, alsdann die durch die Hemmung der Fußspitze am starren Hinder- 
nisse erzeugte Bewegung von vorne nach hinten. Das Resultat beider Gewalteinwirkungen mußte eine extreme Plantarflexion sein.

Ganz ähnlich war die Entstehung der Luxation in dem von Füster an erster Stelle beschriebenen Falle (6): Ausgleiten auf dem Erdboden, Fall mit voller Wucht nach rückwärts, Anprall der Fußsohle bei schräger Haltung des Fußes an ein starrwandiges Hindernis. In beiden letztgenannten Fällen fand offenbar ein Zusammenwirken von indirekter und direkter Gewalt (Sto ) statt, welches besonders auch für die Fälle maßgebend ist, in welchen die Luxation durch Rückwärtsstürzen bei untergeschlagenem Unterschenkel entsteht. Ein besonders deutliches Beispiel hierfür ist der Fall Schanz (4): Patientin rutschte eine Treppe herab und kam am Fuße derselben so zu Falle, daß sie sich auf den untergeschlagenen linken Fuß setzte, der mit der Spitze hängen geblieben und so in Plantarflexion geraten war.

Entsprechend dürfte der Vorgang bei Füsters (6) an 2. Stelle erwähnten Patientin gewesen sein, von der es in der Krankengeschichte heißt, daß sie „über eine Stiege herabgestürzt sei, wobei ihr Fuß unter ihren Körper zu liegen kam“.

Daß auch bei vorneüberstürzendem Körper die Verletzung zustande kommen kann, ist einleuchtend, wenngleich bisher anscheinend seltener beobachtet. Abweichend von den übrigen Autoren gibt Le s er (8) diese Entstehungsart als die einzige an, indem er sagt, daf für das Zustandekommen der Luxation nach hinten ein Fall (aus bedeutender Höhe), bei dem der Fuß mit seinem Rücken den Fußboden zuerst erreicht und dadurch übermäßig plantarflectiert wird, maßgebend sei. Ich habe in der mir zugänglichen Literatur Beispiele hierfür nicht auffinden können; dagegen dürfte dor von mir veröffentlichte Fall hier anzuführen sein, bei welchem die Patientin ganz bestimmt angibt, vornüber bei gestrecktem Körper abgestürzt zu sein. -

Der Luxations-Mechanismus ist im Genaueren nach Wilhelm Henke (11) folgender : vermöge der übermäßigen Plantarflexion bildet sich am hintern Ende der Talusrolle für die Tibia ein Hebelstützpunkt; das Gelenk klafft vorn, die gespannte Kapsel birst und die nach vorne gerichtete Componente der fortwirkenden Kraft stößt die Tibia durch den Kapselriß hindurch auf den vorderen Abschnitt des Talus. (In einem von W en del (1) citierten Falle (Hanmann) stand die Tibia sogar auf dem Os naviculare.) Hier stemmt sie sich bei der vollkommenen Luxation vor der Rolle fest, sobald der Fuß wieder seine rechtwinklige Stellung zum Unterschenkel einzunehmen sucht.

Nicht selten bleibt noch ein teilweiser Contact der correspondie- 
Ein Beitr. zur Kenntnis d. traumat. Luxationen des Fußes im Talocruralgel. 257

renden Gelenkflächen bestehen; es kommt dann wie in unserem Falle zur incompleten Luxation (Subluxation).

Im Momente der Luxation entstehen ausgedehnte Bänderzerreißungen. Nach den von Hönigseh mied (15) u. a. vorgenommenen Leichenversuchen zerreilen in jedem Falle die Hemmungsbänder der forcierten Plantarflexion: das Ligament. talo-tibiale anter. und das Ligam. talo-fibulare anter., meist auch das Ligam. deltoides (in 70 Proz. der Versuche Hönigschmieds kam es zu einer Zerreißung sämtlicher Bandverbindungen zwischen $F u ß$ und Unterschenkelknochen).

Von der Ausdehnung der Zerreilungen hängt im besondern das Verbalten der Fibula ab. Sind dieselben zwischen ihrem untern Ende und Fuß vollständig, so rutscht die Fibula in toto mit der Tibia nach vorn; sind dieselben dagegen nur geringfügig, dann kann es entweder - wie im Falle Engelhardt (5) und im Falle Bulley - (cit. bei Wendel (1)) zu der äußerst seltenen Entstehung einer Luxation des Fußes mit der (intakten) Fibula nach hinten kommen, oder letztere bricht in ihrem untern Drittel, ihr unteres Fragment rückt mit dem Fuße - gewissermaßen als ein Anhang desselben - nach hinten, ihr oberes (welches mit dem unteren meist einen nach hinten offenen stumpfen Winkel bildet) folgt der Tibia nach vorn. Das Vorhandensein dieser Fraktur der Fibula ist nach dem modernsten Standpunkte der Wissenschaft für die Mehrzahl der Fälle anerkannt, vgl. H of f a (16) und Handbuch der prakt. Chirurgie (17).

In den mir aus der Literatur zugänglichen Fällen habe ich dieselbe allerdings nur $3 \mathrm{mal}$ ausdrüeklich erwähnt gefunden: Fall Gönı̈ry (3), Fall Schanz (4) und bei einer Patientin der v. Bergmannschen (17) Klinik; nicht ausgeschlossen - wenn auch nicht bestimmt nachgewiesen - erscheint ihr Vorhandensein im Fall I F üsters.

Ein Beispiel für ihr Vorkommen auch bei incompleter Luxation ist der von mir veröffentlichte Fall, in dem auch die typische (von Dupuytren coup de hache genannte (cit. bei Gömöry (3)) Knikkung an der Frakturstelle vorhanden war.

Hinsichtlich ihres Zustandekommens erscheint mir Füsters (6) Ansicht sehr annehmbar, wonach die Fraktur nur als sekundär entstanden zu denken ist und zwar (in Übereinstimmung mit $\mathrm{Krön}$ leins (13) Auffassung) „durch Fortdauer der Gewalt auf die unbeweglich fixierte schon luxierte Tibia, die dann infolge ihrer abnormen Stellung die fest mit ihr verbundene Fibula fracturiert."

Recht häufig sind auch Brüche am innern oder äußern Knöchel im Momente der Luxation, welche in Übereinstimmung mit dem Er- 
gebnisse der Hönigschmiedschen (13) Versuche als wahre Abrißfracturen anzusehen sein dürften.

Engelhardts (5) Patient hatte einen solchen Abrißbruch am innern Knöchel, bei Füsters (6) einer Patientin bestand dieselbe Complication sogar im Bereiche beider Knöchel. Schanz (4) gibt in seinem Falle die Wahrscheinlichkeit einer Fractur am innern Knöchel zu (,es scheint vom innern Knöchel ein Stück abgebrochen und im ursprunglichen Zusammenhange geblieben zu sein.“) Göschels (2) Fall ist in dieser Hinsicht - zumal von ihm kein Röntgenbild vorhanden ist - nicht ganz aufgeklärt trotz G's Angabe: „eine Knöchelfractur kann nicht nachgewiesen werden."

Eine recht wichtige Complication ist ferner eine gleichzeitige Weichteilverletzung mit Eröffnung der Haut, wodurch die Luxation zu einer sogen. "offenen" wird, "sei es daß die Tibia die Haut durchstößt, sei es daß Quetschung und Zerrung der Haut nachträglich zur Gangrän führt" (Lossen (11)). Während Lossen (11) das Vorkommen dieser Complication für selten hält, sind Hoffa (16) und Nasse-B orchardt,(17) der entgegengesetzten Ansicht. - W endel (1) führt 6 Fälle aus der Literatur an, in denen 5 mal sich Weichteilzerreißungen bis in das Gelenk erwähnt finden, die allein durch von außen direkt einwirkende Gewalt entstanden waren; die Wunde saß dann ausschließlich am Fußrücken. In einem 6. Falle (Schinzinger) - Sprung aus einer fahrenden Droschke auf den Fuß - war eine Querwunde am Fußrücken und an der Außenseite. Die Fibula sah heraus.

Ein Beispiel für die oben erwähnte Möglichkeit, daß nachträglich Hautgangraen durch Zerrung oder Quetschung entstehen kann, ist ein weiterer Fall bei Wendel (1) citiert: (Middeldorpf): Keine Wunde und Fraktur angegeben bei einer Subluxation (17.jähriger Mann); vor der Reposition, die erst nach 5 Tagen erfolgte, bestand starke Compression der Venen. Gangraen der Haut. Phlegmone. Exitus.

Sieht man von diesen Complicationen $a b$, so dürften im allgemeinen die klinischen Erscheinungen der Luxation sehr charakteristisch sein. Der vordere Teil des Fußes ist verkürzt, der Fersenteil um ebensoviel verlängert („die Ferse weit nach hinten gedrängt." Fall Göschel (2)), starkes Vorspringen des Fersenhöckers (Fall Fü ster II (6)). Auffallende Vergrößerung des anter. poster. Durchmessers des Fußgelenks" (Fall Schanz (4)). Vorn springt auf dem Fußrücken die Tibia vor, deren scharfrandige Gelenkfläche zum Teil unter der in einer "gespannten Falte" (16) herüberziehenden (16) Haut durchgefüblt werden kann (Fall Göschel (2), Schanz (4), Engelbardt (5), Füster I (6)) - und zwar um so mehr, je tiefer die Fußspitze steht. 
Ein Beitr. zur Kenntnis d. traumat. Luxationen des Fufes im Talocruralgel. 259

Hinten springt die Achillessehne vor, die in einem nach hinten offenen Bogen zum Fersenhöcker zieht und zwischen sich und den Unterschenkelknochen, bezw. Tibia eine tiefe Rinne freilält (Fall Fü ster I (6), Gömöry (3)).

Die Sehnen der Zehenstrecker sind durch die Tibia gehoben und ziehen als gespannte Stränge über den Fußrücken (f. Fall Gömöry (2)) Der Fuß steht entweder gerade nach vorn oder leicht abduciert (Lossen (11)), Fußspitze „zeigt nach auswärts" (Fall Schanz) (4) in "mäBiger Valgusstellung" (Fall der Marburger Klinik). Recht erbeblich um seine senkrechte Achse gedreht war der Fuß im Falle Gömöry (2), wo eine Außenrotation der Fußspitze um ca. 700 stattgefunden hatte als Ausdruck der secundären Lagerung des Fußes im Sinne Henkes (cit. bei Gömöry (3)), wonach der Fuß nach stattgehabter Verletzung in jeder Mittellage stehen bleiben kann. - Diese eigenartige Combination von Luxation nach hinten mit Eversion (vgl. (20) Knust), war schon Mal gaigne bekannt, welcher sie als Luxation nach innen und vorn bezeichnete, indem ihm für die Namengebung das Verhalten der Unterschenkelknochen als des eigentlich luxierten Körperteiles maßgebend war (vgl. Gömöry (3)).

Die Fußsohle sieht gewöhnlich nach unten, diese Stellung des Gelenkes in Plantarflexion war erheblich im Falle Gömöry (3), wo die Fußspitze um ca. 450 über die rechtwinklige Fußstellung hinaus gesenkt war, geringgradig bei Göschels Patienten und in dem Falle der Marburger Klinik, garnicht vorhanden bei Engelhardts (5) Patienten, dessen Fuß im "rechten Winkel zum Unterschenkel“ stand. Der Fuß ist in seiner Stellung meist (durch Bänder- und Kapselspannung); fixiert, die Plantar- und Dorsalflexion entweder aufgehoben oder nur in geringem Umfange ausführbar - wenigstens in frischen Fällen. Daß bei länger bestehenden (veralteten) Luxationen eine verhältnismäßig ausgiebige Beweglichkeit vorhanden sein kann, zeigt der Fall Schanz (4) (5 Monate alte Verletzung), wo die Beweglichkeit der Hälfte der Norm gleich kam.

Von Gurlt (12) wird als regelmäßiges Symptom eine große Beweglichkeit des Fußes, eine leichte Hin- und Herschiebbarkeit des Fußes in sagittaler Richtung angegeben. Durch Leichenversuche ist festgestellt, daß nach beiderseitjger Zerstörung der Bandverbindungen diese abnorme Beweglichkeit einen so hohen Grad erreichen kann, daß der Fuß sich wie in einem Schlittenapparat nach vorn und hinten verschieben läßt. 


\section{Diagnose.}

Die Diagnose gilt fast allgemein als leicht, und es dürfte tatsächlich Fälle geben, wo dieselbe schon durch die bloße Inspection gestellt werden kann auf Grund der veränderten Längenverhältnisse von Vorder- und Fersenteil des Fußes und der Wahrnehmung des „stufenförmigen" Vorsprunges der Tibia am Fußrïcken. -

Die Schwierigkeit der Diagnose wird um so größer sein, je erheblicher die Weichteilschwellung und je weniger vollständig die Luxation ist, da in letzterem Falle die Symptome manchmal nur eben angedeutet vorhanden sein können.

Differentialdiagnostisch kann in solchen unklaren Fällen in Betracht kommen: die sehr seltene Luxatio pedis sub talo poster., bei welcher jedoch das Talocruralgelenk frei beweglich ist und beide Mal. leolen ihre Lage zum Talus nicht geändert haben, auch könnte wohl einmal die etwas weniger seltene Luxation des Talus nach vorn in Frage gezogen werden. Doch ist hierbei der Vorderfuß verlängert, und die Malleolen sind dem Boden genähert; auch springt der Talus sehr deutlich als harter Körper am Fußrücken oder an einem der Seitenränder hervor.

Diese theoretisch sehr einfach erscheinenden Unterscheidungsmomente am Lebenden festzustellen, ist bei starkem Bluterguß und der meist vorhandenen großen Schmerzhaftigkeit eine äußerst schwierige Aufgabe, und unter solchen Umständen dürfte selbst eine Untersuchung in Narkose nicht immer zu einem ganz sichern bezw. richtigen Ergebnisse führen. In derartigen Fällen tritt der Segen der Röntgendurchleuchtung dann so recht zu Tage, wie in besonders lehrreicher Weise aus der Krankengeschichte meines Falles hervorgeht.

Auch in dem 2. Falle Füsters (6) sind allem Anschein nach die (durch sehr starke Weichteilschwellung verursachten) Schwierigkeiten der Diagnose erst durch die Aktinographie überwunden worden.

Daß auch bei diagnostisch in der Hauptsache klaren Fällen zur Erkennung bezw. Sicherstellung von complicierenden Frakturen das Röntgenverfahren gar nicht entbehrt werden kann, geht aus Fall I Füster (6), Fall Engelhardt (5) und Fall Schanz (4) hervor: in beiden ersteren wurde erst so eine Fraktur am Malleolus internus erkannt, in letzterem eine Fibulafraktur (von der Spuren schon vorher bemerkt worden waren) in unzweifelhafter Weise festgestellt.

An dieser Stelle erscheint es angebracht, den Fingerzeig Nass eBorchardts (17) als sehr beherzigenswert zu empfehlen, laut dessen auf Grund einer eigenen Erfahrung es als unbedingt notwendig em- 
Ein Beitr. zur Kenntnis d. traumat. Luxationen des Fußes im Talocruralgel. 261

pfohlen wird, das Fußgelenk mindestens von zwei Seiten zu durchleuchten, da sonst eine Fibulafractur übersehen werden könne. Zum Beweise dafür wird eine Beobachtung aus der v. Bergmannschen Klinik angeführt, d. i. Fall V meiner Zusammenstellung, bei welcher in einem durch Beleuchtung des Fußgelenks von vorn nach hinten gewonnenen Röntgenbilde die Fibula intact erschien, während bei seitlicher Durchleuchtung eine Fractur derselben mit Sicherheit nachgewiesen wurde.

Nicht uninteressant dürfte hier die Feststellung sein, daß unter den mit Röntgenstrahlen untersuchten Fällen - soweit mir solche in der Literatur zugänglich waren - sich bisher kein einziger gefunden hat, bei dem nicht irgend eine complicierende Fractur vorhanden wäre; dasselbe gilt von meinem Falle aus der Marburger Klinik.

Wie für die Diagnose, so ist auch für die Prognose dank der Aktinographie und der immer zunehmenden Popularität der letzteren ein wesentlicher Umschwung zum Bessern nicht zu verkennen. Erstens hat die Prognose an sich an Exactheit natïrlich ungemein gewonnen, und zweitens ist sie meines Erachtens dadurch gebessert, daß nunmehr Verkennungen der Luxation bezw. zu späte Reposition kaum noch vorkommen dürften, bezw. doch immer seltener werden, während sie früher doch nicht so ganz selten waren (in 15 Prozent der Fälle bis 1898).

Einige recht lebrreiche Beispiele aus Wendels (1) Zusammenstellung verdienen hier angeführt zu werden. Im Fall Boy er war (12) Reposition nach 1 Monat unmöglich. Ausgang in Ankylose. Bei Polaillons Patientin (Nr. 36) gelang die Reposition nach 5 Wochen nicht vollständig; erst nach 2 Monaten vermag Patientin ohne Beschwerden zu gehen. Noch übler erging es dem Patienten Ansselins (Nr. 33) bei dem sich die unblutige Reposition nach 8 Tagen unmöglich erwies und der - trotz operativen Eingriffs - erst nach 9 Wochen imstande war, ohne Krücken zu gehen.

Am verhängnisvollsten waren die Folgen einer nicht rechtzeitig erfolgten Reposition im Falle Mid del d or p fs (Nr. 29), obwohl der unbenutzt verstrichene Zeitraum hier geringer war als bei den vorigen Fällen - (er betrug nämlich nur 5 Tage) - nnd obwohl die Reposition gelungen zu sein scheint. Es trat infolge starker Compression der Venen Gangraen der Haut, Phlegmone und Exitus nach drei Monaten ein.

Ausdrücklich hervorgehoben sei, daß es sich in diesen 2 letzteren Fällen um Subluxationen handelte, deren Prognose doch im allgemeinen als günstig gilt. (Lossen (11)). Bei der vollkommenen Luxa- 
tion ist wegen der häufigen complicierenden Fracturen und der häufig starken Weichteilverletzung die Prognose stets mit Vorsicht zu stellen. Sehr leicht kann eine teilweise Versteifung des Fußes zurückbleiben.

Nach Vidal soll eine große Neigung der eingerichteten Luxation zu Recidiven - infolge des Zuges von seiten der Wadenmuskeln vorhanden sein (H of a (16)). Wird die Luxation verkannt, - vgl. oben - so tritt die Versteifung meist in Spitzfußstellung ein, wodurch die Gebrauchsfähigkeit natürlich sebr beeinträchtigt ist (Fall I F üst ers) (6). (9 Wochen nach der Verletzung vermag die Pat. nur mühsam sich mit Hilfe zweier Stöcke kleine Strecken fortzubewegen); Fall Sch a n z (4) (gut 5 Monate alte Verletzung): „Gang läßt viel zu wünschen übrig, benutzt einen Stock und hinkt erbeblich".

Wie auch in diesen scheinbar ungünstigen Fällen noch ein verbältnismäßig gutes Resultat erzielt wurde, wird später ausgeführt werden. Daß es für den Ausgang jeder Luxation außer der rechtzeitigen Erkennung und Reposition von größter Wichtigkeit ist, ob eine offene oder subcutane (und subcutan bleibende) Verletzung vorliegt, bedarf keiner weiteren Auseinandersetzung.

\section{Behandlung.}

Die Therapie hat bei frischen Fällen die möglichst baldige Reduction des Fußes auf rationellem Wege zu erstreben. Forcierte Plantarflexion, Zug am Fuße nach vorn bei gleichzeitigem Druck auf den im Knie flectiert gehaltenen Unterschenkel nach hinten, zum Schluße Dorsalflexion bilden das typische Repositionsverfahren.

In Fällen unvollkommener Luxation - wie aus dem meinigen zu ersehen ist - genügt manchmal schon ein bloßer Zug am Fuße nach abwärts und gleichzeitiger Druck auf das untere Unterschenkelende in der Richtung von vorn nach hinten.

Im Falle Gömöry (3) soll sogar ein „leichter Zug" am Fuße genügt haben.

Die Anwendung der Narkose dürfte im allgemeinen nicht zu entbehren und möglichst in Form des Ätherrausches anzuwenden sein. In der Wendel'schen (1) Arbeit finden sich hinsichtlich etwa angewandter Narkose bei den einzelnen Fällen keine Angaben außer bei dem von Wendel selber veröffentlichten Falle der Tübinger Klinik (aus dem Jahre 1896), bei welchem die Reposition in Narkose herbeigeführt wurde, ebenso ist die Anwendung der Narkose ausdrücklich erwähnt im Falle Göschel (2), Engelhardt (5); sie wurde auch in meinem Falle angewandt. Der Erfolg der Repositionsversuche war in allen ganz frischen Fällen ein günstiger außer bei 
Engelhardts (5) Patienten, wo die Reposition nicht vollkommen gelang. Besondere Sorgfalt und Mühe erfordert die Behandlung frischer complicierter (offener) Luxationen. Bei diesen wird man nach gründlicher Reinigung des Gliedes und des vorspringenden Knochens die Reposition ausführen (welche in 5 derartigen Fällen Wendels (1) (Nr. 22-26) gelungen ist). ,Die Wunde ist nicht zu nähen, sondern durch Tamponade offen zu halten. Macht die Reposition Schwierigkeiten, so muß die Wunde erweitert werden, sollte auch dann die Reposition nicht gelingen, so muB man von dem vorspringenden Knochen ein Stuck resecieren" - wie es im Falle Schinzingers, cit. bei Wendel (1), wo die Fibula aus der Wunde herausstand, geschah - und in jedem Falle von zu starker Beschmutzung des hervorstehenden Knochens zu geschehen hat.

Wenn das Gelenk nachträglich vereitert, dann kommt die secundäre Resection - meistens nur eine partielle des Fußgelenkes - in Frage. Sie gibt gewöhnlich quoad vitam und quoad functionem recht gute Resultate und macht in der Regel eine Amputation überflüssig (17), die in der vorantiseptischen Aera nur allzu häufig erforderlich wurde. Die Nachbehandlung der in Rede stehenden Fußluxation, auch wenn sie mit keiner Hautwunde compliciert ist, hat mit großer Vorsicht zu geschehen, einmal wegen der meist ausgedehnten Zerreißung des Bandapparates, sodann wegen der so überaus häufigen Com. plication mit einer Fractur.

In rechtwinkliger Stellung zum Unterschenkel wird der $\mathrm{FuB}$ in der Mitte zwischen Adduction und Abduction mehrere Wochen (je nach dem Grade der Verletzung am Bandapparat und Skelett 4-6 Wochen) im Schienen- oder Hartverbande fixiert. Sodann läßt man Massage und passive Bewegungen (leichtere medicomechanische Übungen) folgen; das Tragen eines gut sitzenden Schnürstiefels ist für die Folgezeit noch recht zweckmäßig.

Weit schwieriger als die bisher ins Auge gefaßten frischen Fälle lassen sich im allgemeinen die älteren bezw. veralteten Fälle behandeln. Ich habe aus der mir zu Gebote stehenden Literatur den Eindruck gewonnen, daß - mit Ausnahme des 1. Falles von Füster (6) die unblutige Therapie schon nach verhältnismäßig kurzem Bestande (vergl. Fall Ansselin (8 Tage alt), beide cit. bei Wendel (1), Fall Boyer (1 Monat alt), machtlos war, und kann nicht finden, daß die Angabe mancher Autoren, z. B. v. Nasse und Borchardt (17), es scheine, daß veraltete Luxationen sich noch nach ziemlich langer Zeit unblutig reponieren lassen, eine nennenswerte Stütze aus den bisher veröffentlichten Fällen finde. Allerdings habe ich bereits einen 
Ausnahmefall anerkannt: Füster (6) hat nämlich in neuerer Zeit ein Verfahren veröffentlicht, welches bei einer 9 Wochen alten Luxation laut Röntgenbild mit guten Erfolge in der Grazer Klinik durch v. Hacker angewandt worden ist, es bestand in Anlegung des Lorenzschen Osteoklast-Redresseurs an den Unterschenkel derart, daß in Narkose durch Riemenzug am Fußrücken zunächst eine energische Plantarflexion zustande kam, dann wurde das Gelenk gewaltsam (durch Händekraft) mobilisiert, und während der Fuß in extreme Plantarflexion gedrängt wurde, wurde durch $\mathrm{Zug}$ an einem um den Calcaneus herumgelegten Riemen der Fuß in toto nach vorn gebracht, so daß darauf die bisherige Deformität des Gelenkes völlig geschwunden war und sich damit die Reposition als gelungen erwies.

Dieses ein Beispiel für unblutige Reposition eines veralteten Falles. lbr steht, im Falle sie auch nach der Tenotomie der Achillessehne mißlingen sollte, die blutige Reposition gegenüber (Fall Ansselin, cit. bei Wendel (1)), zu der sich eventuell die Durchmeißelung der schief geheilten Fracturen gesellt, oder die Resection des Gelenks kommt in Frage. Nicht für nötig befunden - wenngleich anscheinend in Erwägung gezogen - wurde das blutige Verfabren von Schanz (4) in seinem 5 Monate alten Falle, in welchem der Repositionsversuch (i. Narkose) mißlungen war. Die Operation wurde durch einen Schienenhülsenapparat ersetzt, durch welchen die an sich ziemlich schlechte Function des Fußes ganz erheblich gebessert sein soll.

Faßt man den Erfolg der Behandlung in den in der Literatur veröffentlichten Fällen ins Auge, so muß ich Füster (6) Recht geben, wenn er in den diesbezüglichen Mitteilungen "fast gar keine besonders erfreulichen Resultate" zu finden vermag.

Unter den von Wendel (1) zusammengesteliten 26 Fällen laßt kaum die Hälfte in unzweifelhafter Weise den Eindruck völlig wiederhergestellter Gebrauchsfähigkeit des Gelenkes erkennen (Fall $14,16,17,18,20,23,28,30,31,32,37$ ), welche allerdings besonders bei dem blutig reponierten Falle Ansselin anzuerkennen ist. „Patient ging nach 9 Wochen frei, und schließlich wurde ein bewegliches Gelenk erzielt". Unter den 6 offenen Luxationen Wendels ist nur eine (Fall 23) mit Erzielung guter Brauchbarkeit des Fußes angegeben.

Besonders ungünstig war unter den veralteten Fällen: Boyer (12), cit. bei Wendel (1) - Reposition nach 1 Monat versucht Ausgang in Ankylose; ferner Fall Polaillon, cit. bei Wendel (1), in welchem nach 5 Wochen trotz Tenotomie der Achillessehne sich die normalen Gelenkverhältnisse nicht wiederherstellen ließen. Nicht 
günstig war auch in einem Falle der neuesten Zeit (Engelhardt) (5) der Ausgang, obgleich die Verletzung frisch zur Reposition kam Letztere gelang nicht vollständig, und die nachträglich aufgetretene Vereiterung eines wohl bei der Reposition entstandenen Blutergusses führte hier zur Ankylose des Gelenkes. Einen recht günstigen Verlauf nahm das Heilverfahren bei Gömörys (3) Patienten, der schon in der 2. Woche im Verbande Gehversuche mit bestem Erfolge machte und dessen Gelenk nach 6 Wochen völlig normale Conturen und Freisein der passiven Beweglichkeit zeigte. Der Ausgang vom Falle Göschel (2) ist mir leider nicht bekannt geworden. Bei ihm war die Extremität nur 8 Tage im Hartverbande, dann Massage.

Als recht befriedigend dürfte in dem von mir beobachteten Falle der Heilerfolg zu bezeichnen sein. Nach gut 4 Wochen konnte Patientin bereits ohne Verband leidlich gehen und wurde nach 1 weiteren Woche mit wenig eingeschränkter Beweglichkeit des Fußgelenkes und hinreichendem Gebvermögen bei guter Stellung des Gelenkes und der Fibula entlassen. Als günstiges Beispiel für den Ausgang einer veralteten Luxation nach ihrer Reposition ist nochmals der bereits citierte Fall 1 Füster (6) zu erwähnen, dessen Patientin bereits nach ca. 2 Wochen im Gypsverbande recht befriedigende Gehversuche zu machen vermochte und 6 Monate nach ihrer Entlassung mitteilte, dal3 Stellung und Beweglichkeit des Fußes ebenso vollkommen normal geworden sei, als sie es vor der Verletzung gewesen seien. Unter Berücksichtigung der Möglichkeit durch das von $F u ̈$ ster beschriebene Verfahren mit dem Lorenzschen Instrumente die Reposition unter genauer Dosierung der Kraftentfaltung nach der heute allgemein üblichen rationellen Methode vornehmen zu können, sollte man meines Erachtens in keinem Falle von veralteter Fußluxation einen Versuch mit diesem Hilfsmittel verabsäumen, bevor man zu einem blutigen Eingriffe schreitet. Der II. Fall F゙üsters läßt sich hinsichtlich der Therapie leider hier nicht verwerten, da seitens der Patientin die Vornahme der Reposition nicht gestattet wurde.

Nunmehr am Schlusse der Besprechung angelangt, fasse ich das Endergebnis derselben kurz dahin zusammen, daß

1. die Entstehung der Luxation durch Ein- bezw. Mitwirkung direkter Gewalt nicht so selten zu sein scheint, wie man bisher angenommen hat.

2. Für die Diagnose dank dem Röntgenverfahren eine neue Ära begonnen hat in der Hinsicht, daß auf Grund der bisher bekannt gewordenen Röntgenbilder der Zweifel, ob es wirklich ganz reine, d. h. 
mit gar keiner Fractur complicierte Luxationen des Fußes n. b. gibt '), nicht unbegründet erscheint.

3. Infolge der immer größer werdenden Einbürgerung der Röntgendiagnostik die Prognose durch rechtzeitige Diagnose in Zukunft immer besser werden dürfte, wobei ganz besonders die Subluxationen in Betracht kommen.

1. Die auf diesem Gebiete in der neuesten Zeit erzielten Fortschritte für das Arbeitsfeld der Unfallheilkunde von ganz besonders hohem praktischen Werte sein dürften.

\section{Literaturverzeichnis.}

1) Wendel, Die traumatischen Luxationen des Fußes im Talaruralgelenk (v. Bruns Beiträge zur klin. Chirurgie. Bd. XXI, Heft 1).

2) $\mathrm{G}$ öschel, Über traumatische Luxationen der unteren Extremität (Münchener medizinische Wochenschrift 1896, No. 18).

3) Gö möry, Über die traumatischen Luxationen des Fußes im Talocruralgelenk (Wiener medizinische Wochenschrift 1902, No. 17-20).

4) Schanz, Ein Fall von Iuxation des Fußes nach hinten. v. Langenbecks Archiv 1902, Band 68, Heft 2.

5) Engelhardt, Eine seltene Fußgelenksluxation. Deutsche Zeitschrift für Chirurgie, Band 69.

6) Füster, C̈ber einen Fall von Luxatio pedis posterior inveterata und; deren Reposition. v. Bruns Beiträge zur klin. Chirurgie 1904, Bd. XLII, Heft 3.

7) Koenig, Lehrbuch der speziellen Chirurgie. 6. Aufl. 1894.

8) Leser, Die spezielle Chirurgie. 3. Aufı 1897.

1) Eine Stütze dürfte dieser Zweifel ferner an zahlreichen in obigen Sinne verwertbaren Befunden erhalten, welche zum Teil bei blutiger Reposition irreponibler Luxationen, zum Teil durch Röntgendurchleuchtung (vgl. Handbuch der prakt. Chirurgie [17]) an anderen großen Gelenken (vorwiegend am SchulterHüft- und Ellenbogengelenk), in Form von Abriß- und Absprengungsfracturen gewonnen sind.

Siehe v. Bruns Beiträge zur klin. Chirurgie:

Band XV, Januar 1896: H. Reerink, Zur operativen Behandlung irreponibler Luxationen.

Band XVII, Januar 1897: G. Drehmann, Zur operativen Behandlung irreponibler traumatischer Hüftgelenksluxationen.

Und eben dort: J. Finckh, Über Reponibilität der veralteten Schultergelenkluxationen.

Band XX, März 1898: F. Cuhorst, Zur operativen Behandlung irreponibler Luxationen im Ellenbogengelenk.

Band XXIX: E. Schoch, Die irreponiblen Schulterluxationen und ihre Behandlung.

Centralblatt für Chirurgie 1900: R. Bunge, Zur operativen Behandlung der veralteten irreponiblen Luxation im Ellenbogengelenk. 
Ein Beitr. zur Kenntnis d. traumat. Luxationen des Fußes im Talocruralgel. 267

9) Albert, Lehrbuch der speziellen Chirurgie. 5. Aufl. 1898.

10) Helferich, Atlas und Grundriß der traumatischen Frakturen und Luxationen. 6. Aufl. 1903.

11) Lossen, Artikel „Fufgelenksluxationen“ in Encyklopädie der gesamten Chirurgie. Herausgeg. v. Kocher u. d e Quervain. 1902.

12) Gurlt, Derselbe Artikel in Eulenburgs Realencyklopädie der gesamten Heilkunde. 3. Aufl. Band VIII, 1895.

13) Krönlein, Lehre von deu Luxationen. Deutsche Chirurgie, Lieferung 26.

14) Lo ssen, Verletzungen der untern Extremitāt. Deutsche Chirurgie, Lieferung 65.

15) Hönigschmied, Leichenexperimente über die Zerreißungen der Bănder im Sprunggelenk mit Rücksicht auf die Entstehung der indirekten Knöchelfracturen. Deutsche Zeitschrift für Chirurgie $187 \pi$, Band 8.

16) $\mathrm{H}$ of a a, Lehrbuch der Fracturen und Luxationen. 4. Aufl. 1904.

17) Nasseu. Borchardt, Artikel Luxationen des Fußes im 'Talocruralgelenk, in Handbuch der praktischen Chirurgie (v. Bergmann-Bruns-Miculicz) 1903.

18) Centralblatt für Chirurgie, Sämtliche Jahrgänge von 1879 bis einschl. 1904.

19) Schultz, Zur Kasuistik seltener traumatischer Luxationen der untern Extremität. Monatschrift für Unfallheilkunde 1898, No. 9.

20) Knust, Uber Fußgelenksluxation durch Rotation nach außen. (Eversion.) In.-Diss. Greifswald 1897. 